Reprod. Nutr. Dévelop., 1987, 27 (3), 641-648.

\title{
Action des cations bivalents sur l'activité de la phosphatase alcaline des microvillosités intestinales. Influence du pH et du substrat
}

\author{
Sylviane TARDIVEL, Francine RABESONA, Léa LANDIHARINTSOA, Yvonne \\ DUPUIS
}

Laboratoire du Métabolisme minéral des Mammifères (EPHE), Physiologie, Faculté de Pharmacie, 92290 Chatenay-Malabry.

Summary. Effect of bivalent cations on alkaline phosphatase activity of intestinal microvilli. Influence of $\mathrm{pH}$ and substrate.

At alkaline or neutral $\mathrm{pH}$ and with PNPP or ATP as substrates, the effect of cations on alkaline phosphatase of jejunal microvilli was shown to differ, i.e. $\mathrm{Mg}^{++}$had an activating effect at high concentrations, $\mathrm{Zn}^{++}$slightly inhibiting, $\mathrm{Ca}^{++}$and $\mathrm{Co}^{++}$were inactive at all concentrations. At neutral $\mathrm{pH}$ with PNPP as a substrate, $\mathrm{Mg}^{++}$and $\mathrm{Co}^{++}$were active, $\mathrm{Ca}^{++}$inactive and $\mathrm{Zn}^{++}$slightly inhibiting at high concentrations. Using ATP as a substrate, all four cations were activators at all concentrations studied $(0,2$ to $5 \mathrm{mM})$. In the latter physiological conditions $(\mathrm{pH}$ and substrate), similar effects of the cations were observed with microvilli from other segments of rat small intestine.

\section{Introduction.}

Pour le dosage de l'activité des phosphatases alcalines, les conditions choisies sont fort éloignées d'un fonctionnement naturel. Par commodité, le substrat couramment employé est le paranitrophénylphosphate (PNPP), le PNP issu de l'action hydrolasique de l'enzyme donnant en milieu sodique une coloration jaune directement mesurable. Le pH retenu 9,8 correspond au maximum d'activité de l'enzyme.

Une électrophorèse des protéines de la microvillosité intestinale montre sur la même préparation que les protéines phosphorylables possèdent les mêmes propriétés électrophorétiques que les phosphatases alcalines (Dupuis et al., 1981; Crouzoulon et al., 1983). L'étude des propriétés biochimiques des protéines phosphorylées de la microvillosité duodénale conduit aussi, après dissociation, à les identifier aux intermédiaires phosphorylés des monomères de la phosphatase alcaline (De Jonge et al., 1981).

Sans préjuger du mécanisme de la phosphorylation de la phosphatase membranaire, on peut retenir que l'activité phosphatasique et la capacité de se phos- 
phoryler constituent deux aspects fonctionnels de l'enzyme. Pour tenter de les relier il faudrait que les deux réactions soient observées dans les mêmes conditions, ce qui n'est pas le cas quand l'activité phosphatasique est mesurée avec le PNPP à pH 9,8 et la phosphorylation avec I'ATP à la neutralité. La mesure des variations d'activité phosphatasique en fonction du $\mathrm{pH}$ et du substrat est l'un des objectifs de ce travail.

On sait l'importance de la phosphorylation des ATPases membranaires dans le transfert des cations. Tandis qu'elles font usage de I'ATP en se phosphorylant, leur activité enzymatique est activée par le cation transféré et ce sont alors des ATPases $\mathrm{Na}^{+} \mathrm{K}^{+} \mathrm{Mg}^{++}$ou $\mathrm{Ca}^{++}$dépendantes. Du fait que les phosphatases alcalines sont aussi des protéines microvillositaires phosphorylables dans quelle mesure peut-on les rapprocher des ATPases ? Elles sont aussi des métalloenzymes. II peut donc être intéressant de rechercher comment l'activité phosphatasique varie, aux divers $\mathrm{pH}$ et pour les substrats précédemment cités, en fonction de la nature et de la concentration en divers cations du milieu d'incubation. C'est l'autre objectif de ce travail qui vient en préambule de l'étude d'une action de ces mêmes cations sur la phosphorylation des protéines microvillositaires.

\section{Matériel et méthodes.}

Produits chimiques. - PNPP (p-nitrophénylphosphate), ATPNa ${ }^{2}$ (Adénosine 5' triphosphate disodique), Elon (sulfate de p-méthyl aminophénol), HEPES (Acide $\mathrm{N}$-2-hydroxyéthylpipérazine- $\mathrm{N}^{\prime}$-2-éthane sulfonique et Tris-(hydroxyméthyl)aminométhane sont des produits Sigma. Les autres réactifs sont des produits Prolabo « Normapur ».

Réactif animal. - Les études sont faites sur des rats adultes Wistar de 3 à 4 mois recevant le régime d'élevage de Randoin et Causeret (1947) qui renferme pour $100 \mathrm{~g}, 600 \mathrm{mg}$ de $\mathrm{Ca}$ et $500 \mathrm{mg}$ de $\mathrm{P}$. L'intestin grêle est prélevé, lavé dans du $\mathrm{NaCl} 9 \%$ et partagé en trois parties : le duodénum du pylore à l'angle de Treitz, le reste de l'intestin est coupé en deux, la partie proximale est appelée " jéjunum " et la partie distale "iléum ". Les trois parties sont fendues longitudinalement et les muqueuses sont séparées par grattage et homogénéisées. Toutes les opérations sont faites à $4{ }^{\circ} \mathrm{C}$.

Préparation des microvillosités. - Les membranes microvillositaires sont préparées à partir d'un homogénat de muqueuse dans un tampon Tris 2 mM-Mannitol $50 \mathrm{mM} \mathrm{pH} \mathrm{7,1} \mathrm{selon} \mathrm{la} \mathrm{méthode} \mathrm{de} \mathrm{Schmitz} \mathrm{et} \mathrm{al.} \mathrm{(1973)} \mathrm{qui} \mathrm{utilise} \mathrm{une} \mathrm{précipita-}$ tion au $\mathrm{CaCl}_{2}$, des centrifugations différentielles ainsi qu'un gradient discontinu de glycérol. Les membranes sont prélevées, lavées et conservées dans le tampon et stockées à $-80^{\circ} \mathrm{C}$.

Contrôle enzymatique. - Les protéines sont dosées par la méthode de Bradford (1976). Les enzymes retenues comme marqueurs de la membrane microvillositaire 
sont l'invertase, la leucyl aminopeptidase et la phosphatase alcaline dosées respectivement selon Dahlquist (1964), Tuppy et al. (1962) et par la méthode de Bessey modifiée (1946).

Les contaminations par les autres fractions cellulaires sont recherchées en mesurant l'activité de la $\left(\mathrm{Na}^{+}-\mathrm{K}^{+}\right)$-ATPase, de la NADPH-cytochrome $\mathrm{C}$ réductase, de la succinodeshydrogénase, de la $\beta$-glucuronidase et de la lactate deshydrogénase comme précédemment décrit (Crouzoulon et al., 1983).

Action des cations sur l'activité phosphatasique. - Cette action est étudiée à pH 7,2 et 9,8 quand le substrat est le PNPP $5 \mathrm{mM}$ et à $\mathrm{pH} 7,2$ et 9,0 quand le substrat est I'ATP. A pH 7,2 le tampon utilisé est un tampon maléate $50 \mathrm{mM}$ ajusté avec $\mathrm{NaOH}$. Les $\mathrm{pH}$ alcalins sont obtenus en tampon $\mathrm{Na}_{2} \mathrm{CO}_{3}-\mathrm{HNaCO}_{3} 30 \mathrm{mM}$.

L'hydrolyse spontanée de l'ATP étant d'autant plus forte que le $\mathrm{pH}$ est plus alcalin, l'essai est réalisé à $\mathrm{pH}$ 9. $\mathrm{Ce} \mathrm{pH}$ a d'ailleurs été utilisé pour des mesures d'activité ATPasique (Wass et Butterworth, 1972 ; Majeska et Wuthier, 1975).

Les cations employés sous forme de chlorure sont le calcium, le cobalt, le magnésium et le zinc dont les concentrations choisies sont données sous les figures.

Les temps d'incubation à $37^{\circ} \mathrm{C}$ varient de 10 à 30 minutes.

Si le substrat est le PNPP, le PNP libéré est mesuré à $405 \mathrm{~nm}$ après arrêt de la réaction par de la soude $0,1 \mathrm{~N}$. Si le substrat est l'ATP, c'est le phosphate libéré qui est dosé par la méthode de Lebel et al. (1978) après arrêt de la réaction par addition d'acide trichloracétique à $10 \%$ et centrifugation à $500 \mathrm{~g}$. Chaque essai est réalisé sur 4 prises de la même préparation.

\section{Résultats.}

\section{I. - Qualité des préparations microvillositaires.}

Le degré de purification est du même ordre dans les différentes parties de l'intestin et il varie sensiblement selon l'enzyme marqueur considéré : il est de 32 pour l'invertase, de 19 pour la phosphatase alcaline et de 15 pour la leucyl-aminopeptidase. Ces différences s'expliquent si l'on considère que l'invertase est strictement localisée dans la bordure en brosse alors qu'une fraction de la phosphatase alcaline et de la leucyl-aminopeptidase se trouve pour la première dans les membranes baso-latérales et dans le cytosol pour la seconde.

Les contaminations par d'autres fractions cellulaires sont pratiquement négligeables dans ce type de préparation. Cependant, un certain niveau d'activité $\left(\mathrm{Na}^{+} \mathrm{K}^{+}\right)$ATPasique indique la présence de membranes baso-latérales. Mais, par rapport aux homogénats de muqueuse, le rapport des enrichissements respectifs en $\mathrm{Na}^{+} \mathrm{K}^{+}$ATPase et en invertase montre que les membranes baso-latérales représentent $1 / 20^{\circ}$ environ des protéines présentes dans la préparation des membranes microvillositaires (Dupuis et al., 1981). 
II. - Activités phosphohydrolasiques des microvillosités jéjunales.

1) En fonction du substrat et $d u p H$. - Les résultats sont présentés sur le tableau 1. Une préparation de microvillosités jéjunales a une activité phosphatasique à l'égard du PNPP environ 7 fois plus forte au pH optimal $(9,8)$ qu'à la neutralité. La même préparation microvillositaire présente en milieu alcalin environ 15 fois plus d'activité hydrolasique à l'égard de l'ATP dans les conditions choisies d'hydrolyse enzymatique de ce substrat ( $\mathrm{pH} 9$ ) qu'à la neutralité. Rappelons qu'à pH 9,8, I'hydrolyse chimique de I'ATP ne permet pas l'essai d'une activité enzymatique.

TABLEAU 1

Activités phosphohydrolasiques des membranes microvillositaires jéjunales.

\begin{tabular}{ccc}
\hline Substrat & $\mathrm{pH}$ & $\mu$ moles $\mathrm{Pi} / \mathrm{mn} / \mathrm{mg}$ protéine \\
\hline PNPP $5 \mathrm{mM} \ldots \ldots \ldots \ldots \ldots \ldots$ & 9,8 & $16,6 \pm 1,4\left(^{*}\right)$ \\
ATP $5 \mathrm{mM} \ldots \ldots \ldots \ldots \ldots \ldots$ & 7,2 & $2,5 \pm 0,2$ \\
& 9,0 & $22,6 \pm 2,1$ \\
& 7,2 & $1,5 \pm 0,1$ \\
\hline
\end{tabular}

(*) Moyenne $\pm \mathrm{Sm}(\mathrm{n}=4)$.

Activité déterminée à $37^{\circ}$, en tampon maléate $50 \mathrm{mM}$ à pH 7,2 ; en tampon carbonate-bicarbonate $30 \mathrm{mM}$ à $\mathrm{pH} 9,0$ et 9,8 .

2) Effet des cations bivalents. - D'importantes variations dans les activités selon les préparations nous ont conduit, dans l'étude de l'effet des cations, à rapporter à 100 l'activité hydrolasique mesurée sans addition de cations.

L'action de ces derniers est alors calculée à partir de cette base.

a) Substrat PNPP. La figure 1 fait état des résultats fournis par les 4 prépara-

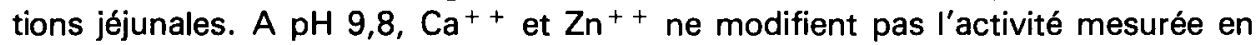
l'absence de cations. On note une action de $\mathrm{Co}^{++}$, légère, mais non significative. La seule augmentation significative d'activité est donnée par la plus forte conc zntration de $\mathrm{Mg}^{++}(2 \mathrm{mM})$.

A pH 7,2, $\mathrm{Ca}^{++} \mathrm{n}^{\prime}$ active toujours pas l'hydrolyse du PNPP. $\mathrm{Zn}^{++} \mathrm{n}^{\prime}$ agit pas non plus et même pour les plus fortes concentrations ( 2 à $5 \mathrm{mM}$ ), on observe un effet inhibant de $\mathrm{Zn}^{++}$, à la limite de la significativité. En ce qui concerne la concentration la plus élevée, $\mathrm{Co}^{++}$et $\mathrm{Mg}^{++}$augmentent de façon significative $(\mathrm{p}<0,05)$ l'activité de la phosphatase.

b) Substrat ATP. A pH 9, les cations se montrent différents dans leur action. Ainsi, $\mathrm{Ca}^{++}$est sans effet, $\mathrm{Mg}^{++}$à 0,5 et $1 \mathrm{mM}$ augmente l'activité hydrolasique des microvillosités jéjunales à l'égard de ce substrat $; \mathrm{Co}^{++}$est aussi activant, mais $\mathrm{Zn}^{++}$, aux trois concentrations employées, diminue, mais non significativement, l'activité hydrolasique (fig. 2).

Ce n'est qu'à la neutralité que les quatre préparations de microvillosités jéjunales ont leur aptitude à hydrolyser l'ATP significativement augmentée à toutes les concentrations étudiées des quatre cations. Le fait retient davantage l'atten- 


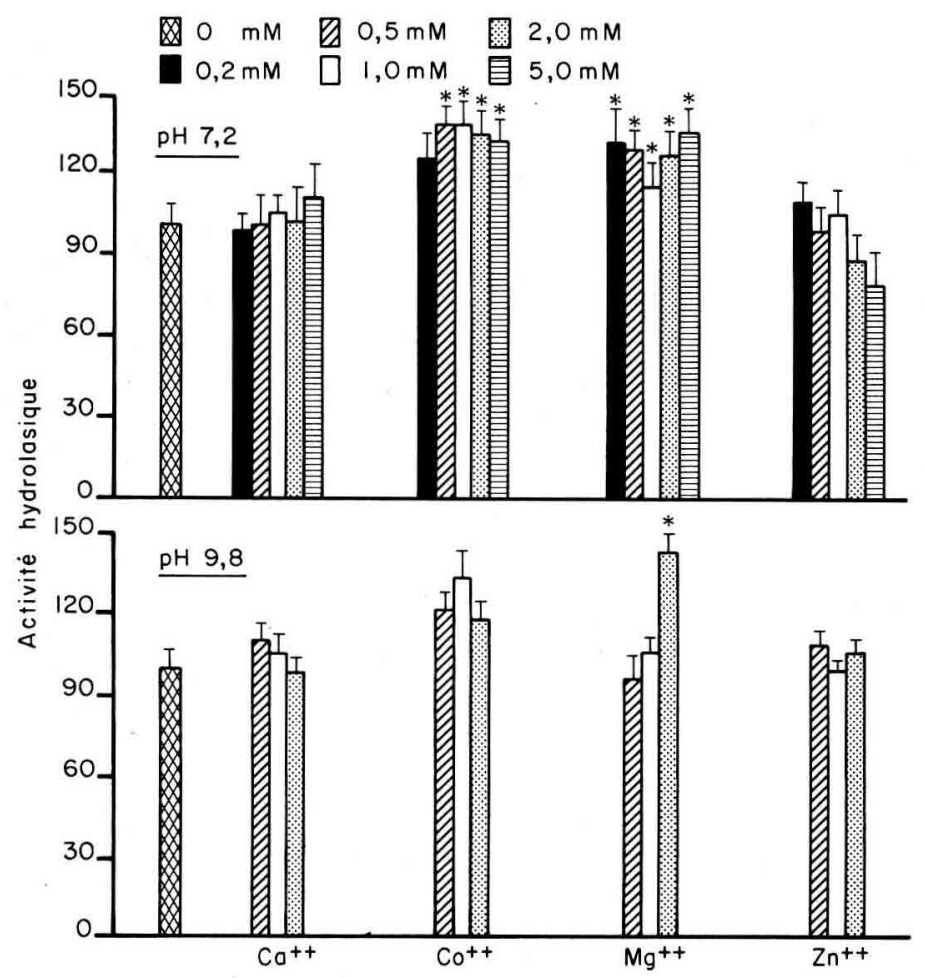

FIG. 1. - Action des cations sur l'activité phosphohydrolasique des membranes microvillositaires jejunales en présence de PNPP $5 \mathrm{mM}$, tampon maléate $50 \mathrm{mM}, \rho H 7,2$ ou carbonate-bicarbonate $30 \mathrm{mM}, \mathrm{pH} 9,8$.

Les résultats sont exprimés en p. 100 par rapport au témoin. ${ }^{*} \mathrm{p} 0,05 ;{ }^{*}$ p 0.01 .

tion pour $\mathrm{Ca}^{++}$et $\mathrm{Zn}^{++}$qui, dans tous les cas précédents, $\mathrm{n}^{\prime}$ ont montré aucun effet notable. En général, l'activation de l'hydrolyse de I'ATP, déjà manifeste à la plus faible concentration $(0,2 \mathrm{mM})$ est plus marquée quand augmente la concentration en cation. Cependant, pour $\mathrm{Zn}^{++}$, on observe plutôt l'effet inverse, significativement plus faible à mesure que sa concentration croît au-delà de $1 \mathrm{mM}$ (fig. 2).

III. - Effets des cations sur l'activité phosphohydrolasique des microvillosités duodénales, jéjunales, iléales.

L'effet des quatre cations est recherché à la neutralité au moyen de l'ATP sur des microvillosités provenant des trois régions de l'intestin grêle. Comme précédemment, les valeurs portées dans le tableau 2 concernent 4 préparations différentes, les résultats obtenus sans adjonction de cation étant rapportés à 100 . Dans ces conditions, on note que les quatre cations activent I'hydrolyse de l'ATP 


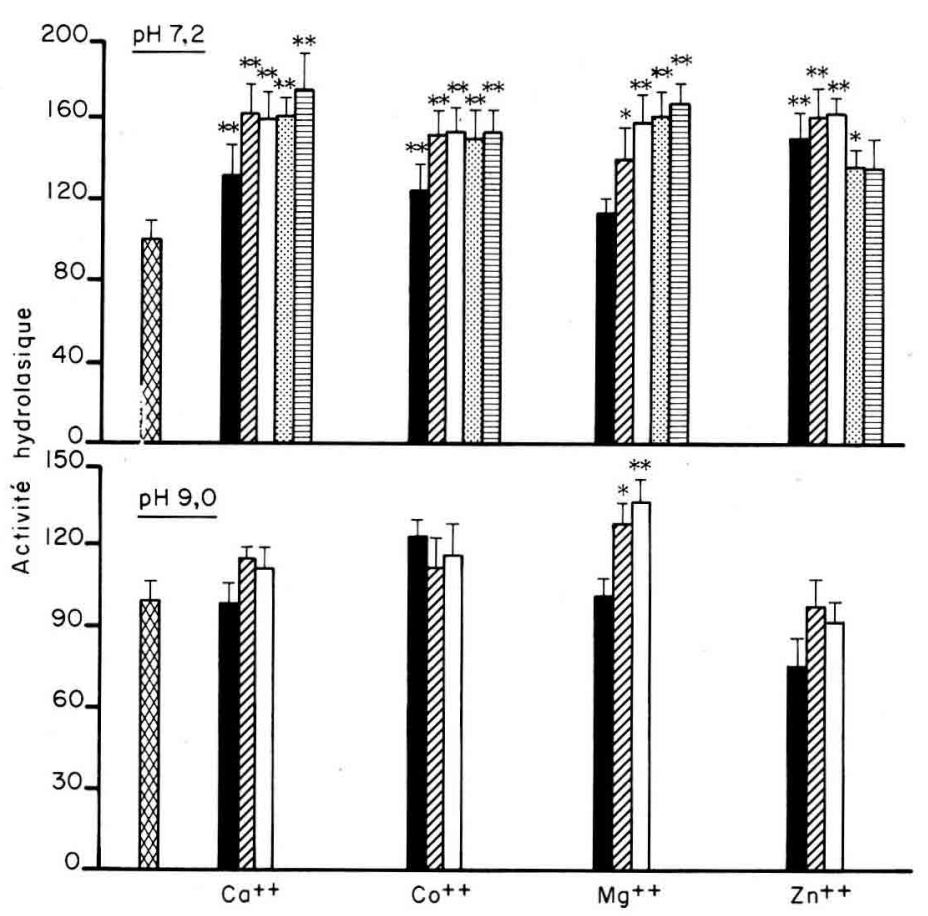

FIG. 2. - Action des cations sur l'activité phosphohydrolasique des membranes microvillositaires jéjunales en présence d'ATP $5 \mathrm{mM}$, tampon maléate $50 \mathrm{mM}, \mathrm{pH} 7,2$ ou carbonate-bicarbonate $30 \mathrm{mM}, \mathrm{pH} 9,0$.

Les résultats sont exprimés en p. 100 par rapport au témoin. ${ }^{*} p<0,05 ;{ }^{*}$ p $<0,01$.

TABLEAU 2

Action des cations sur l'activité phosphohydrolasique, à l'égard de l'ATP, de microvillosités intestinales.

\begin{tabular}{lccccc}
\hline \multicolumn{1}{c}{ Microvillosités } & 0 & $\mathrm{Ca}^{++}\left(^{*}\right)$ & $\mathrm{Co}^{++}$ & $\mathrm{Mg}^{++}$ & $\mathrm{Zn}^{++}$ \\
\hline Duoodénum $\ldots \ldots \ldots \ldots \ldots \ldots 100 \pm 18\left(^{* *}\right)$ & $232 \pm 35$ & $207 \pm 18$ & $215 \pm 28$ & $175 \pm 21$ \\
Jéjunum $\ldots \ldots \ldots \ldots \ldots \ldots \ldots 100 \pm 21$ & $234 \pm 16$ & $173 \pm 23$ & $183 \pm 18$ & $160 \pm 16$ \\
lléum $\ldots \ldots \ldots \ldots \ldots \ldots \ldots \ldots 100 \pm 11$ & $189 \pm 27$ & $153 \pm 33$ & $166 \pm 27$ & $161 \pm 19$ \\
\hline
\end{tabular}

(*) $1 \mathrm{mM}$ (en cation).

(**) Pourcentage de variation par rapport aux témoins. Moyenne $\pm S m(n=4)$. Activité déterminée à $37^{\circ}$ en tampon maléate $50 \mathrm{mM}$ pH 7,2, ATP $5 \mathrm{mM}$.

pour toutes les préparations microvillositaires. La nature du cation ou la provenance de la microvillosité n'interviennent pas de façon décisive sur cette activation. On peut toutefois noter que $\mathrm{Ca}^{+}{ }^{+}$se montre le plus efficace et que la microvillosité iléale est celle dont l'activité ATPasique est la moins influencée par les cations. 


\section{Discussion.}

Cette étude confirme des faits classiquement connus. $\mathrm{Mg}^{++}$augmente l'activité de la phosphatase alcaline, présentement dans des conditions variées de $\mathrm{pH}$ et de substrat ; $\mathrm{Co}^{++}$possède certaines des aptitudes de $\mathrm{Mg}^{++}$, il pourrait remplacer $\mathrm{Zn}^{+}$, cation constitutif de plusieurs phosphatases alcalines de mammifères. II pourrait également se substituer au $\mathrm{Mg}^{++}$en tant qu'activateur de la fonction hydrolasique (Cathala et al., 1975 et Harkness, 1968). $\mathrm{Zn}^{++}$est parfois inhibant (Sugiura et al., 1977). Un effet activant de cations bivalents, dont $\mathrm{Ca}^{++}$, a déjà été rapporté sur des bordures en brosse d'épithélium intestinal dans les conditions conventionnelles, $\mathrm{pH} 9$ et PNPP, de mesure de l'activité phosphatasique (Hanna et al., 1979). Mais cet effet activant des cations peut être attribué à une neutralisation de I'EDTA, complexant utilisé par ces auteurs au cours de la préparation des bordures et lors du prétraitement avant la mesure de l'activité enzymatique. Par contre, une autre étude montre que la plupart des cations bivalents inhibent l'activité de phosphatases alcalines de divers tissus purifées en l'absence de complexant (Rej et Brétaudière, 1980). Dans ce type d'étude l'état cationique initial de l'enzyme est donc déterminant.

Le fait nouveau qui ressort du présent travail est la généralité de l'activation par les cations ${ }^{++}$du pouvoir phosphohydrolasique de la microvillosité intestinale, en milieu neutre, vis-à-vis de I'ATP.

$\mathrm{L}^{\prime}$ action du $\mathrm{Ca}^{++}$sur l'activité hydrolasique tant de la microvillosité entérocytaire que de la phosphatase alcaline a été particulièrement étudiée.

Ghijsen et al. (1980) détectent dans la microvillosité intestinale une CaATPase de faible affinité. Utilisant divers inhibiteurs spécifiques de la Ca-ATPase et de la phosphatase alcaline, ces auteurs montrent que cette activité CaATPasique de la microvillosité est attribuable à une réaction non spécifique de la phosphatase alcaline. En effet, la théophylline $2,5 \mathrm{mM}$ inhibe de façon semblable I'hydrolyse du PNPP et de I'ATP mesurée en présence de $\mathrm{Ca}^{++} 0,2 \mathrm{mM}$ et la chlorpromazine, inhibiteur spécifique des Ca-ATPases, n'influence pas l'hydrolyse des deux substrats étudiés.

Williams et al. (1985) montrent que l'activité PNPPasique d'une phosphatase alcaline hautement purifiée par chromatographie d'affinité et immunoprécipitation est insensible au $\mathrm{Ca}^{++}$tandis que ce cation stimule son activité ATPasique. De plus, ces auteurs n'ont pu séparer des fractions dont le substrat préférentiel aurait été soit l'ATP, soit le PNPP ou soit le phytate, toutes les fractions obtenues hydrolysent ces trois substrats.

Les divers aspects de la question de l'identité et de la spécificité de la phosphatase, de la Ca-ATPase et de la phytase ont été rapportés par Mc Comb et al. (1979).

Dans ce travail nous avons montré d'une part que dans l'étude de l'action des cations sur l'activité hydrolasique de la phosphatase alcaline, l'état cationique initial de l'enzyme est déterminant. D'autre part, le fait nouveau est la généralité de l'activation par les cations ${ }^{++}$du pouvoir phosphohydrolasique de la microvillosité intestinale, en milieu neutre, vis-à-vis de I'ATP. 


\section{Références}

BESSEY Y. O. A., LOWRY O. H., BROCK M. J., 1946. A method for rapid determination of alkaline phosphatase with five cubic millimeters of serum. J. biol. Chem., 164, 321-329.

BRADFORD M. M., 1976. A rapid and sensitive method for the quantitation of microgram quantities of protein utilizing the principle of protein-dye binding. Anal. Biochem., 72, 248-254.

CATHALA G., BRUNEL C., CHAPPELET-TORDO D., LAZDUNSKI M., 1975. Bovine kidney alkaline phosphatase : catalytic properties, subunit interactions in the catalytic process and mechanism of $\mathrm{Mg}^{++}$stimulation. J. biol. Chem., 250, 6046-6053.

CROUZOULON G., DUPUIS Y., REY D., FOURNIER P., 1983. Phosphorylated proteins from rat intestinal microvilli membranes. Electrophoretic similarities with alkaline phosphatase. Mol. Physiol., 4, 323-329.

DE JONGE H. R., GHIJSEN W. E., VAN OS C. H., 1981. Phosphorylated intermediates of $\mathrm{Ca}^{++}$ATPase and alkaline phosphatase in plasma membranes from rat duodenal epithelium. Biochim. Biophys. Acta, 647, 140-149.

DAHLQUIST A., 1964. Method for assay of intestinal dissacharidases. Anal. Biochem., 7, 18-25.

DUPUIS Y., CROUZOULON G., FOURNIER P., 1981. Does the inhibition of microvillus proteins phosphorylation by lysin explain the activity of the latter on calcium transfert? Int. J. Biochem., 13, 1163-1170.

GHIJSEN W. E., DE JONG M. D., VAN OS C. H., 1980. Dissociation between $\mathrm{Ca}^{+}+$ATPase and alkaline phosphatase activities in plasma membranes of rat duodenum. Biochim. Biophys. Acta, 599, 538-551.

HANNA S. D., MIRCHEFF A. H., WRIGHT E. M., 1979. Alkaline phosphatase of basal lateral and brush border plasma membranes from intestinal epithelium. J. Supramol. Str., 11, 451-466.

HARKNESS D. R., 1968. Studies on human placental alkaline phosphatase. 2. Kinetic properties and studies on the apoenzyme. Arch. Biochem. Biophys., 126, 513-523.

LEBEL D., POIRIER G., BEAUDOIN A., 1978. A convenient method for the ATPase assay. Anal. Biochem., 85, 86-89.

Mc COMB R. B., BOWERS G. N., POSEN S., 1979. Alkaline phosphatase, pp. 872-874, Plenum Press, New York.

MAJESKA R. J., WUTHIER R. E., 1975. Studies on matrix vesicles isolated from chick epiphyseal cartilage. Association of pyrophosphatases and ATPase activities with alkaline phosphatase. Biochim. Biophys. Acta, 391, 51-60.

RANDOIN L., CAUSERET J., 1947. Constitution d'un régime alimentaire équilibré pour des jeunes rats blancs destinés au dosage des vitamines $A$ et $D$ par les méthodes biologiques. Bull. Soc. Sci. Hyg. alim., Paris, 35, 14-22.

REJ R., BRETAUDIĖRE J. P., 1980. Effects of metal ions on the measurements of alkaline phosphatase activity. Clin. Chem., 26, 423-428.

SCHMITZ J., PREISER H., MAESTRACCI D., GHOSH B. K., CERDA J. J., CRANE R. K., 1973. Purification of the intestinal brush border membrane. Biochim. Biophys. Acta, 323, 98-112.

SUGIURA M., HIRANO K., IFNO S., SUSUKI H., ODA T., 1977. Effects of $\mathrm{Zn}^{++}$and $\mathrm{Mg}^{++}$on alkaline phosphatase from human placenta and intestin. Chem. Pharm. Bull., 25, 653-656.

TUPPY H., WIESBAUER U., WINTERSBERGER E., 1962. Aminosaüre-p-nitroanilide als substrate für Aminopeptidasen und andere proteolytische Fermente. Hoppe-Seyler's Z. f. Physiol. Chem., 329, 278-288.

WASS M., BUTTERWORTH P. J., 1972. The effect of calcium ions on the ATPase activity of pig kidney alkaline phosphatase. Biochim. Biophys. Acta, 290, 321-326.

WILLIAMS S. A., CULP J. S., BUTLER L. G., 1985. The relationship of alkaline phosphatase, $\mathrm{Ca}^{++}$ATPase and Phytase. Arch. Biochem. Biophys., 241, 10-13. 